\title{
Non-destructive detection of highway hidden layer defects using a ground-penetrating radar and adaptive particle swarm support vector machine
}

\author{
Xinyu Liu ${ }^{\text {Corresp., } 1,2,3}$, Peiwen Hao ${ }^{1}$, Aihui Wang ${ }^{4}$, Liangqi Zhang ${ }^{2}, \mathrm{Bo} \mathrm{Gu}^{3}$, Xinyan Lu ${ }^{3}$ \\ 1 CHANG'AN University, Xian, China \\ 2 Henan Wanli Rord\&Bridge Group Co. Ltd., Xuchang, China \\ ${ }^{3}$ School of Electric Power, North China University of Water Resource and Electric Power, Zhengzhou, China \\ 4 Zhongyuan University of Technology, Zhengzhou, China \\ Corresponding Author: Xinyu Liu \\ Email address: Ixy@ncwu.edu.cn
}

In this paper, a method that uses a ground-penetrating radar (GPR) and the adaptive particle swarm support vector machine (SVM) method is proposed for detecting and recognizing hidden layer defects in highways. Three common road features, namely cracks, voids, and subsidence, were collected using ground-penetrating imaging. Image segmentation was performed on acquired images. Original features were extracted from thresholded binary images and were compressed using the $\mathrm{kl}$ algorithm. The SVM classification algorithm was used for condition classification. For parameter optimization of the SVM algorithm, the grid search method and particle swarm optimization algorithm were used. The recognition rate using the grid search method was $88.333 \%$; the PSO approach often yielded local maxima, and the recognition rate was $86.667 \%$; the improved adaptive PSO algorithm avoided local maxima and increased the recognition rate to $91.667 \%$. 


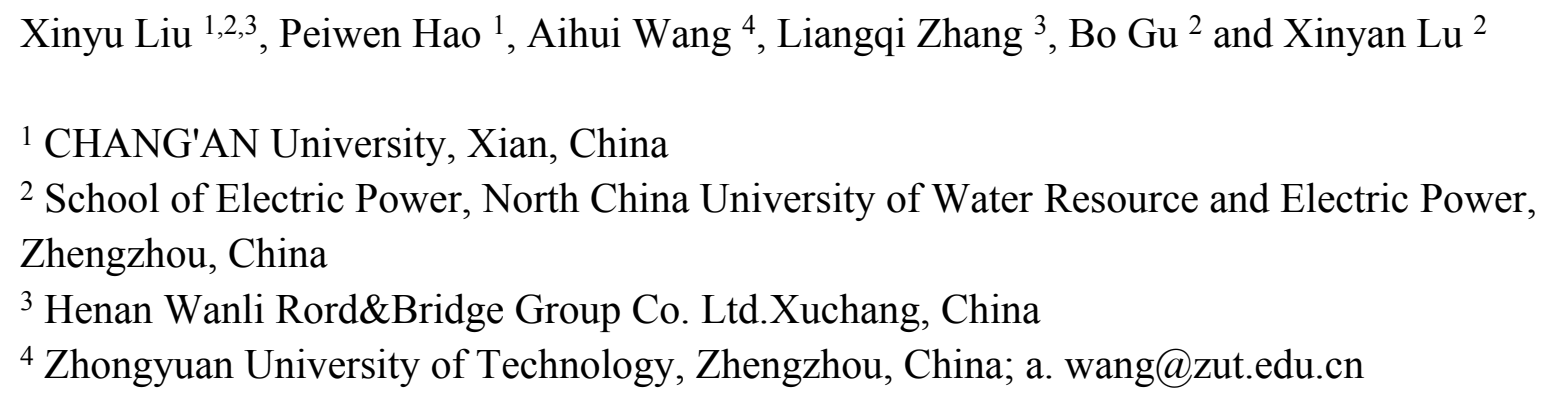

\section{Abstract}

In this paper, a method that uses a ground-penetrating radar (GPR) and the adaptive particle swarm support vector machine (SVM) method is proposed for detecting and recognizing hidden layer defects in highways. Three common road features, namely cracks, voids, and subsidence, were collected using ground-penetrating imaging. Image segmentation was performed on acquired images. Original features were extracted from thresholded binary images and were compressed using the $\mathrm{kl}$ algorithm. The SVM classification algorithm was used for condition classification. For parameter optimization of the SVM algorithm, the grid search method and particle swarm optimization algorithm were used. The recognition rate using the grid search method was $88.333 \%$; the particle swarm optimization (PSO) approach often yielded local maxima, and the recognition rate was $86.667 \%$; the improved adaptive PSO algorithm avoided local maxima and increased the recognition rate to $91.667 \%$.

Keywords: 5 Ground penetrating radar (GPR); Image segmentation; Feature extraction; Support vector machine (SVM); Grid search method; Particle swarm optimization (PSO)

\section{Introduction}

Many forms of road deterioration can develop after prolonged utilization of expressways; examples include crack formation, development of voids, and subsidence [1]. The main cause of these conditions is the appearance of cracks under the roadbed, which gradually affects the road 
38

39

40

41

42

43

44

45

46

47

48

49

50

51

52

53

54

55

56

57

58

59

60

61

62

63

64

65

66

67

68

69

70

71

\section{Image preprocessing and feature extraction} detection methods. testing of road defects based on this. the obtained method performs well in detecting hidden pavement defects.

\section{Detection principle of the ground penetrating radar}

surface and causes surface cracks. Continued use of expressways causes incremental damage and can significantly increase the amount and type of maintenance work. With increasing traffic volumes in China, it is necessary to develop more efficient and automated road condition-

Much research has been conducted on using ground-penetrating radars (GPRs) for landform surveys. With the continuous development of the GPR technology and with the improvement of detection accuracy, the use of the GPR technology for non-destructive detection of structural road conditions has been garnering increasing attention. Yuanlei et al. used the GPR technology to detect different physical anomalies, based on physical simulations and field tests, and the response characteristics across the two scenarios were consistent [2]. Shili et al. used the GPR technology to detect hidden defects, such as road breaks, voids, and subsidence, and acquired defect images [3]. Literature [4] uses amplitude attenuation method in ultrasonic testing to evaluate the corrosion damage of reinforced structures in concrete roads. Document [5] detects the defects in concrete road base by ultrasonic detection technology and establishes the mathematical model of sound wave propagation in different media. Literature [6] establishes the mathematical model of ultrasonic wave propagation in asphalt pavement and concrete pavement to identify highway surface defects, analyzes its feasibility and realizes ultrasonic nondestructive

Using methods from the image recognition field, this study focuses on the design of shallow hidden defect classifiers based on the support vector machine (SVM) algorithm. The SVM method has been widely studied and applied in different contexts. Hou improved the SVM algorithm's low precision around the hyperplane and reduced its computational complexity for processing large amounts of data; they also improved the algorithm's training efficiency, and managed to reduce the number of false calls [7]. El-Saadawi used the SVM algorithm for the stator winding protection of synchronous generators, and achieved good results [8]. The SVM algorithm has been widely used in a variety of context, such as big data, medical, agricultural, and transportation applications [9,10,11]. Using the SVM algorithm, this study optimizes and improves its parameter selection process. By comparing the optimization performance of the grid search (GS) [12] method and the particle swarm optimization (PSO) [13] algorithm, the superiority of the PSO algorithm is demonstrated, and the SVM classification algorithm for PSObased parameter optimization is studied [14]. By collecting radar images of three diseases on the 107 national highway section from Zhengzhou to Xinxiang in Henan Province, it is shown that

In the GPR approach, the ground is irradiated by high-frequency electromagnetic waves using a transmitting antenna, while the waves reflected from the ground are detected using a receiving 
76

77

78 GPR detection process

79 The reflection coefficient of an electromagnetic wave mainly depends on the dielectric constants

80 of the medium in which the wave travels originally and the medium from which the wave is

81 reflected, as given by Equations 1 and 2:

$$
\begin{gathered}
r=\frac{\sqrt{\varepsilon_{1}}-\sqrt{\varepsilon_{2}}}{\sqrt{\varepsilon_{1}}+\sqrt{\varepsilon_{2}}} \\
v=\frac{c}{\sqrt{\varepsilon_{1}}}
\end{gathered}
$$

82 Where, $r$ is the reflection coefficient; $\varepsilon_{1} \quad \varepsilon_{2}$ are the relative dielectric constant of the incident

83

84

85

86

87

88

89

90

91

92

93

94

95

96

97

98

99

100

101

102

103

medium and the medium from which the wave is reflected, $v$ is the echo speed, and $c$ is the speed of light in vacuum.

\section{Selection of the ground penetrating radar}

The ground penetrating radar used in this study is LTD-2100 ground penetrating radar system developed by China Electronics Technology Group Corporation. The main components of the system are $900 \mathrm{MHz}$ shielded antenna, data acquisition host, computer, data cable, etc.

\section{Image preprocessing}

The original images of roads detected by a GPR include the reflection characteristics of road conditions and various types of clutter caused by environmental factors. The clutter distribution is typically non-uniform, affecting the correct recognition and classification of road conditions. To improve the accuracy of the road condition determination, it is necessary to remove the effect of this clutter [16]. To extract the features associated with various road conditions, the acquired images should be segmented.

1) Image filtering

The objective of image filtering is to minimize the effects of noise and interference in raw images. The noise and interference are mainly attributed to the processes - image acquisition and image transmission. Gaussian filtering is typically used for image denoising, which can remove unnecessary interference and protect the edge of the image.

\section{2) Image segmentation}

The objectives of image segmentation are to classify foreground and background pixels, and to determine the organization of foreground pixels (i.e., detect foreground objects) [17]. 
104 The Canny operator has been widely used for image segmentation of radar images, and its

105

106

107

108

109

110

111

112

113

114

115

116

117

118

119

120

121

122

123

124

125

126

127

128

129

130

131

132

133

134

135

136

137

segmentation performance after Gaussian filtering is demonstrated in Figure 2. Fig. 2 (A) is the original radar acquisition diagram. Fig. 2 (B) is the result after Gaussian transformation. It can be seen that the clutter in the original image is removed. Fig. 2 (C) is the disease characteristic waveform diagram extracted after Canny operator. To retain only the necessary information, the image was subjected to the morphological operations of expansion and corrosion; the results are shown in Figure 3. Figure 3 (A) is the image after Canny operator segmented the disease waveform. Figure 3 (B) is an enhanced disease waveform diagram after the segmented waveform is processed by digital morphology, so as to facilitate the extraction of disease features in the following text. The processed image was segmented and objects were extracted using the vertical projection method, as shown in Figure 4.

\section{3) Feature extraction}

Feature extraction should satisfy the following requirements: 1) features should have strong antiinterference ability; 2) features should be insensitive to translation, rotation, and scale transformation of images; 3 ) features should be insensitive to geometric distortions; 4) distance between similar images should be as small as possible; and 5) distance between different images should be as large as possible. The algorithm for extracting feature vectors should be simple, and the dimensionality of the feature space should not be too high for ensuring the classification performance of the system [18]. To satisfy the above requirements, the following features were used: area, image complexity, image texture, and seven rectangular features.

In this study, three types of road hazards are studied, namely, road cracking, hollowing, and subsidence. Through simulation and actual images, combined with the classification of expert experience, three types of samples are collected to describe these conditions. Twentynine feature vectors were extracted for each sample, and the obtained set of features is shown in Table 1, Table 2, Table 3.

The dimensions of the different features are very different. Direct use of feature data not only reduces the system performance, but also affects the classification accuracy. To avoid this shortcoming, it is necessary to perform data normalization [19]. Let the eigenvector of a pattern vector be $X=\left(x_{1}, x_{2}, \ldots x_{m}\right)$. Then the normalized eigenvector $x_{i}^{\prime}$ is

$$
x_{i j}^{\prime}=0.1+\frac{0.8\left(x_{i j}-x_{i, \min }\right)}{x_{i, \max }-x_{i, \min }}(i=1,2, \ldots, m)
$$

Where, $x_{i, \max }, x_{i, \min }$ are the maximum and minimum of $\left\{x_{i}(k) \mid k-1, \ldots, P\right\}$, and $P$ is the overall number of samples in the training set.

4) Feature selection

In this study, $K-L$ transformation was used as the feature selection algorithm [20]. $K-L$ transformation can take into account different classification information and realize supervised

Peer] Comput. Sci. reviewing PDF | (CS-2020:11:55513:1:2:NEW 24 Jan 2021) 
138 feature extraction. Under the criterion of minimum mean square error, it can obtain an 139 orthogonal transformation matrix $A$ that can map the original feature $X$ from high-dimensional 140 space $D$ to low-dimensional space vector $y . K-L$ transformation can retain the data component 141 with the largest variance in the original data and highlight the data difference. Empirical 142 knowledge was used for removing several highly correlated features, and $K-L$ changes were 143 used for reducing the dimensionality of the original feature space. A relatively simple class144 center classifier was used for identifying the samples after the $K-L$ dimensionality reduction 145 transformation. The recognition rate is shown in Figure 5 for the test set.

146 According to Figure 5, when the dimensionality of the feature space reaches 12, the

147 recognition rate saturates; thus, the optimal dimensionality of the feature space is $12 . K-L$ 148 transformation can take into account different classification information, realize supervised 149 extraction of features with too high correlation, and then use $K-L$ transformation to extract 150 data information from them, thus achieving the purpose of compressing dimensions and 151 improving recognition rate.

152

\section{The SVM classifier design}

\section{Basic principle of the SVM}

155

156

157

158

159

160

161

162

163

SVM is a binary classification algorithm that is trained using the supervised learning paradigm. The algorithm attempts to determine an optimal classification hyperplane, whereby the edge distance between two sample classes and the dividing hyperplane (decision boundary) is maximized. The larger the edge distance is, the more separated the two sample classes are, the stronger the classification robustness, and the better the method's generalizability [21]. The hyperplane equation is

$$
\omega^{T} x+b=0
$$

Where $\omega$ is the normal to the hyperplane, and $x$ determines the angle with the hyperplane; $b$ is the distance between the hyperplane and the origin. The hyperplane is denoted as $(\omega, b)$; then, the distance from a certain point (sample) to the hyperplane $(\omega, b)$ is denoted by $r$ :

$$
r=\frac{\left|\omega^{T} x+b\right|}{\|\omega\|}
$$

164 Let the support vector be 1 and point away from the hyperplane, so that $\left|\omega^{T} x+b\right|=1$, and let the 165 vectors in the two different classes be $\gamma$ and point away from the hyperplane: 


$$
\gamma=\frac{2}{\|\omega\|}
$$

166 For optimal segmentation, we need to find the hyperplane with the largest interval; that is, we

167 need to find the parameters $\omega$ and $b$ that maximize $\gamma$. According to equation (6), only $\|\omega\|^{-1}$

168 should be maximized.

\section{2. Optimization of the SVM classifier parameters}

170 We used MATLAB (Mathworks, Inc.) to validate the diagnostic accuracy of the SVM-based 171 classifier with respect to the road conditions. We obtained a dataset comprising 100 examples of 172 road cracking, hollowing, and subsidence conditions; $80 \%$ of these images were used for training 173 the method, while the remaining $20 \%$ were used for testing the classifier.

174 The performance of any SVM classifier depends on the penalty factor and kernel function 175 parameters. The optimal parameter values are typically determined using the grid search 176 approach, which exhausts the set of possible parameter combinations to determine the optimal 177 combination.

178 The penalty term $c$ and the kernal function parameters $g$ were considered to grow exponentially 179 in the $\left[2^{-14}, 2^{14}\right]$ range; at the same time, the step between each cell on the grid was set to 0.5 , that 180 is, the growth time-points were $2^{-14}, 2^{-13.5}, \ldots, 2^{14}$. As shown in Figure 6, the best classification 181 performance was obtained for $\log _{2}(c)=7.60$, best $\log _{2}(g)=-6.60$.

182 As shown in the retrieval results above, the accuracy of $\log _{2}(c)$ between $\left[2^{5}, 2^{10}\right]$ and $\log _{2}(g)$ 183 between $\left[2^{-10}, 2^{-4}\right]$ was relatively good; thus, the step was set to 0.2 in this range, and the 184 parameters were further optimized. The results are shown in Figure 7.

185 According to Figure 7, the best classification was obtained for best $=7$ and best $=-5.2$. These 186 optimal values were used as the SVM classifier parameters for validating its classification 187 performance; then, the accuracy of the classifier with these parameters was characterized. The 188 validation results are shown in Figure 8, and the final validation accuracy rate was $88.333 \%$, the 189 image recognition time is $\mathrm{t}=0.630 \mathrm{~s}$.

190 3. Adaptive mutation particle swarm

191 The results obtained using the grid search approach can meet the desired detection requirements, 192 but because the grid search approach only considers discrete combinations of parameters, the 193 optimal solution can be easily missed. At the same time, the grid search method is exhaustive, 194 and needs to consider all the possible cases; thus, this optimization approach is time-consuming. 195 To improve the accuracy and reduce the computation time, the PSO algorithm is proposed in this 196 section. 
197

198

199

200

201

202

203

204

205

206

207

208

209

210

\section{1) The PSO approach}

PSO simulates a flock of birds, which are modeled as massless particles [22]. Each particle has only two attributes: velocity $v$ and position $x$. Velocity represents the speed of movement, and position represents the direction of movement. Each particle searches for the optimal solution separately in the search space, and records it as the current individual extreme value $P$; the individual extreme values are shared among all the particles in the entire swarm, and the optimal individual extreme value is determined as the current global optimal solution $G$ of the entire swarm of particles. All of the particles in the particle swarm adjust their speeds and positions according to the current individual extremum $P$ found by themselves and the current global optimal solution $G$ shared by the entire particle swarm. The underlying PSO process is relatively simple, and can be divided into the following steps: 1 . initializing the particle swarm; 2 .

evaluating the particles, that is, calculating the fitness values; 3 . searching for individual extrema $P ; 4$. searching for the global optimal solution $G ; 5$. modifying the particles' speeds and positions [23]. The update equations are:

$$
\begin{aligned}
& v_{i d}^{k+1}=\omega v_{i d}^{k}+c_{1} \cdot \operatorname{rand}(0,1) \cdot\left(p_{i d}^{k}-x_{i d}^{k}\right) \\
& +c_{2} \cdot \operatorname{rand}(0,1) \cdot\left(p_{g d}^{k}-x_{i d}^{k}\right) \\
& x_{i d}^{k+1}=x_{i d}^{k}+v_{i d}^{k+1}
\end{aligned}
$$

211 Where $\omega$ is the inertia factor, $c_{1}$ and $c_{2}$ are the acceleration constants, and rand $(0,1)$ are random 212 numbers in the interval $(0,1) . p_{i d}$ denotes $d$ the dimension of the individual extreme value of 213 variable $i \cdot p_{g d}$ represents the dimensionality $d$ of the global optimal solution. $k$ represents the 214 current number of iterations.

215 The following parameter values were used: $c_{1}=2, c_{2}=2$, population size $=20$, maximal number 216 of iterations $=200$, and cross-validation fold $K=5$. The fitness curve for the optimized SVM 217 classifier parameters is shown in Figure 9.

218 After iteration 15, the fitness reached its optimal value. At this point, the classifier accuracy was $21989.197 \%$, the best $c$ was 0.7596 , and the best $g$ was 0.7674 . Although the basic PSO algorithm 220 exhibits a good convergence speed and good optimization performance, it can prematurely 221 converge onto a locally optimal solution; as a result, the population will easily stagnate without 222 external pressure.

223 2) Variation improvement

224 To deal with the premature convergence problem, we utilized the concept of mutations that is 225 often used in genetic algorithms, and incorporated mutations into the PSO framework. For that, 226 we defined a trigger to allow particles to escape locally optimal solutions, thus ensuring a global 227 search [24]. The population fitness variance $\sigma^{2}$ was used for determining whether a local 
228 maximum was reached in the iteration process. The population fitness variance $\sigma^{2}$ was defined 229 as follows:

$$
\sigma^{2}=\sum_{i=1}^{N}\left(\frac{f_{i}-f_{\text {avg }}}{f}\right)^{2}
$$

230

231

232

233

234

235

236

237

238

239

240

241

242

243

244

245

246

247

248

249

250

251

252

253

254

255

256

257

In the above equation, $N$ is the number of particles, $\mathrm{f}$ is the normalized calibration factor, $f_{i}$ is the fitness of the first particle, and $f_{a v g}$ is the average fitness. It can be seen that the larger the value of $\sigma^{2}$, the more divergent the particle swarm; conversely, the smaller the population fitness variance, the more convergent the particle swarm is. Values of $\sigma^{2}$ close to zero indicate that the particle swarm is approaching the globally optimal solution or is converging onto a locally optimal solution. To avoid premature trapping of the particle swarm in local optima, the swarm is subjected to mutations:

$$
x_{i}(k+1)=C \cdot \operatorname{rand}(0,1) \cdot x_{i}(k)
$$

In the above equation, $C$ is a normally distributed random number in the $[0,1]$ interval and is the variation factor; $\operatorname{rand}(0,1)$ is a random number in the $[0,1]$ range; $k$ is the number of iterations. Mutations alter the particles' positions and thus allow to escape local optima [25].

The fitness curve of the improved adaptive mutation PSO algorithm for SVM-based parameter optimization is shown in Figure 10. Evidently, the best fitness converges to a local maximum after 17 generations. Mutations allow the particle swarm to escape this local maximum after 97 generations; after that, optimization parameters are determined with higher accuracy. The finally estimated parameter values are: $c=7.751, g=5.754$. The fitness at this global optimum is $91.698 \%$.

\section{3) Simulation results}

Based on the results in the previous section, the SVM model was constructed using the following parameter values: $c=0.7596$ and $g=0.7674$, which were determined using the PSO method.

The results are shown in Figure 11.

For these parameter values, the accuracy of the model was $86.667 \%$, the image recognition time is $t=0.590 \mathrm{~s}$; however, the recognition accuracy had not improved. The main explanation was the trapping of the particle swarm in a local optimum during the process of parameter optimization. An SVM classifier was then constructed and validated using $c=7.751$ and $g=5.754$; these parameter values were determined using the adaptive mutation PSO algorithm. The validation results are shown in Figure 12.

From Figure 12, the classification accuracy of the SVM classification model with the parameter values determined by the adaptive mutation PSO was $91.667 \%$, the image recognition time is $\mathrm{t}=$ 
258 259

260

261

262

263

264

265

266

267

268

269

270

271

272

273

274

275

276

277

278

279

280

281

282

283

284

285

286

287

288

289

290

291

292

293

$0.615 \mathrm{~s}$. This classification accuracy is significantly higher compared with that achieved by the SVM method that uses parameters optimized by the grid search approach.

\section{Conclusions}

According to the requirements of automatic recognition of highway hidden layer conditions, this paper proposes an automatic detection and recognition method that uses an SVM with parameters optimized using the adaptive mutation PSO approach. In this method, PSO with mutations is used for parameter optimization. In this study, three different methods were used for parameter optimization: 1) the grid search method, 2) the PSO approach, and 3) the adaptive mutation PSO. MATLAB and Python were used for implementing these optimization methods, and the optimization processes and their results were validated. Compared with the grid search method and the simple PSO approach, the accuracy of the SVM with parameters optimized using mutation PSO was higher, translating into better performance on automatic identification of highway conditions. Our simulation results showed that the classification accuracy of the SVM classifier with the grid search method was $88.333 \%$, the classification accuracy of the SVM classifier with PSO was $86.667 \%$, and the classification accuracy of the SVM classifier with mutation PSO was $91.667 \%$. Thus, the effect of SVM classifier with mutation PSO is obviously better than that of the other two. Compared with the grid search method, the classification accuracy was improved. However, owing to the pre-processing of images and processing of feature-related data, some defect-related information is likely to become distorted, which can affect the accuracy of recognition. If a zero-distortion image processing method can be found, the recognition accuracy will be greatly improved. At present, only cracks, voids and subsidence can be analyzed and studied, while asphalt pavement diseases and defects can be divided into 11 categories and 21 items. In order to better improve the scope of the identification system, further research should be done on other types of defects.

\section{References}

1. Marecos V, Solla M, Fontul S, Antunes V. Assessing the pavement subgrade by combining different non-destructive methods. Construction and Building Materials, 2017, 135:76-85.

2. Yuan-lei SI, Guo-lin W. Study on the response characteristics of different physical anomalies in the detection of ground-penetrating radar. Journal of Yunnan University (Natural Sciences Edition), 2018, 40(05):903-908.

3. Shi-li GUO, Lei XU. LI, Xiu-zhong. Application of GPR in detection of road surface settlement. Progress in Geophysics, 2018, 33(03):1213-1217.

4. Ming-Te Liang,Po-Jen Su.Detection of the corrosion damage of rebar in concrete using impact-echo method Cem Concr Res,31(2001), pp.1427-1436. 
294 5. Tirupan Mandal,James M.Tinjum,Tuncer B.Edil.Non-destructive testing of cementitiously 295 stabilized materials using ultrasonic pulse velocity test[J]. Transportation Geotechnics, 2016, 6: 296 97-107.

297 6. Wen, M. Balasingam, T. Edil, J. Tinjum, A. Gokce, J. Wang,et alCharacterization of 298 cementitiously stabilized layers for use in pavement design and analysis Project 04-36 Test 299 Procedure Evaluation Report National Cooperative Highway Research Program, 300 Washington,D.C. (2011)

301 7. Hou K, Shao G, Wang H, et al. Research on practical power system stability analysis 302 algorithm based on modified SVM. Protection and Control of Modern Power Systems, 2018, 303 3(1):1-7.

304 8. El-Saadawi M, Hatata A. A novel protection scheme for synchronous generator stator 305 windings based on SVM. Protection and Control of Modern Power Systems, 2017, 2(1):24.

306 9. Wang W, Du X, Wang N. Building a cloud IDS using an efficient feature selection method 307 and SVM. IEEE Access, 2019, 7:1345-1354.

308 10. Wang C, Zhang Y, Song J, et al. A novel optimized SVM algorithm based on PSO with 309 saturation and mixed time-delays for classification of oil pipeline leak detection. Systems 310 Science and Control Engineering, 2019, 7(1):75-88.

311 11. Hongtao Z, Yuxia HU, Hanping M. Image recognition and classification of the stored-grain 312 pests based on support vector machine. Journal of Agricultural Mechanization Research, 2008, 313 08:36-38.

314 12. Xiaosheng L, Zhibang Z. Parameter optimization of Support Vector Machine based on 315 improved grid search method. Journal of Jiangxi University of Science and Technology, 2019, 316 40(01):5-9.

317 13. Yang L, Zhichuan Z, Alin H, et al. Pulmonary nodule recognition based on multiple kernel 318 learning support vector machine-PSO. Computational and Mathematical Methods in Medicine, 319 2018, 2018:1-10.

320 14. Ziji M, Yanru D, Hongli L, et al. Method of forecasting non-equal interval track irregularity 321 based on improved grey model and PSO-SVM. IEEE Access, 2018, 06:34812-34818.

322 15. Benedetto A, Tosti F, Bianchini Ciampoli LB, D’Amico F. An overview of ground323 penetrating radar signal processing techniques for road inspections. Signal Processing, 2017 , 324 132:201-209.

325 16. Bu F, Bu F. A high-order clustering algorithm based on dropout deep learning for 326 heterogeneous data in cyber-physical-social systems. IEEE Access, 2017, PP(99), 6:1-1.

327 17. Fengjun C, Chenghan W, Mengmeng GU, YANDong Z. Spruce image segmentation 328 algorithm based on fully convolutional networks. Transactions of the Chinese Society for 329 Agricultural Machinery, 2018, 49(12):188-194 + 210. 
330 18. Zhiyun X, Hong LIU. Adaptive features fusion and fast recognition of potato typical disease 331 images. Transactions of the Chinese Society for Agricultural Machinery, 2017, 48(12):26-32.

332 19. Chowdhury SA, Stepanov EA, Danieli M, Riccardi G. Automatic classification of speech 333 overlaps: Feature representation and algorithms. Computer Speech and Language, 2019, 55:145334167.

335 20. Jun N. A data reduction algorithm based on K-L feature compression for cloud computing. 336 Microelectronics and Computer, 2016, 33(02):125-129.

337 21. Yueting L, Xiaoxia L, Sixuan L. Imbalanced dataset classification algorithm based on 338 improved support vector machine. Journal of Shihezi University (Natural Science), 2018, 339 36(05):637-643.

340 22. Hsieh YZ, Su MC, Chen JH, et al. Developing a PSO-based projection algorithm for a 341 porosity detection system using X-ray CT images of permeable concrete. IEEE Access, 2018, 342 6:64406-64415.

343 23. Yang J, Wang X, Bauer P et al. Extended PSO Based Collaborative Searching for Robotic 344 Swarms With practical constraints. IEEE Access, 2019, 7:76328-76341.

345 24. Yifei T, Meng Z, Jingwei L, et al. Research on intelligent welding robot path optimization 346 based on GA and PSO algorithms. IEEE Access, 2018, 6:65397-65404.

347 25. Deng W, Yao R, Zhao H, et al. A novel intelligent diagnosis method using optimal LS-SVM 348 with improved PSO algorithm. Soft Computing, 2019, 23(7):2445-2462. 


\section{Table $\mathbf{1}$ (on next page)}

Gray level co-occurrence matrix characteristics of cracks, voids, and subsidence. 
1

\begin{tabular}{ccccc}
\hline Defect type & \multicolumn{5}{c}{ crack } \\
\hline GLCM & $0^{\circ}$ & $45^{\circ}$ & $60^{\circ}$ & $90^{\circ}$ \\
contrast & 1.333 & 2.149 & 1.436 & 2.194 \\
correlation & 0.895 & 0.826 & 0.893 & 0.826 \\
energy & 0.724 & 0.708 & 0.728 & 0.702 \\
homogeneity & 0.988 & 0.962 & 0.972 & 0.967 \\
\hline Defect type & \multicolumn{5}{c}{ void } \\
\hline GLCM & $0^{\circ}$ & $45^{\circ}$ & $60^{\circ}$ & $90^{\circ}$ \\
contrast & 0.998 & 1.501 & 1.273 & 1.806 \\
correlation & 0.473 & 0.235 & 0.335 & 0.075 \\
energy & 0.644 & 0.594 & 0.626 & 0.579 \\
homogeneity & 0.891 & 0.865 & 0.886 & 0.849 \\
\hline Defect type & \multicolumn{5}{c}{ subsidence } \\
\hline GLCM & $0^{\circ}$ & $45^{\circ}$ & $60^{\circ}$ & $90^{\circ}$ \\
contrast & 3.813 & 6.486 & 5.447 & 5.924 \\
correlation & 0.414 & 0.019 & 0.164 & 0.104 \\
energy & 0.795 & 0.750 & 0.768 & 0.758 \\
homogeneity & 0.931 & 0.884 & 0.902 & 0.838 \\
\hline
\end{tabular}




\section{Table 2 (on next page)}

Differential statistical matrix characteristics of cracks, voids, and subsidence. 
1

\begin{tabular}{ccccc}
\hline GLDS & mean & contrast & asm & ent \\
\hline crack & 0.048 & 289.831 & 0.916 & 0.261 \\
void & 0.141 & 660.909 & 0.589 & 2.186 \\
subsidence & 0.121 & 765.796 & 0.794 & 0.5202 \\
\hline
\end{tabular}

2 


\section{Table 3(on next page)}

Seventh order invariant moment feature. 
1

\begin{tabular}{cccc}
\hline $\begin{array}{c}\text { seven-order } \\
\text { Hu moment }\end{array}$ & crack & void & subsidence \\
\hline Ф1 & 5.076 & 4.059 & 4.463 \\
Ф2 & 10.591 & 8.547 & 9.925 \\
Ф3 & 22.646 & 15.286 & 19.998 \\
Ф4 & 22.974 & 15.286 & 22.229 \\
Ф5 & 45.786 & 30.563 & 19.562 \\
Ф6 & 28.343 & 19.562 & 28.508 \\
Ф7 & 43.134 & 35.275 & 38.163 \\
\hline
\end{tabular}

2

3 
Figure 1

GPR detection process.

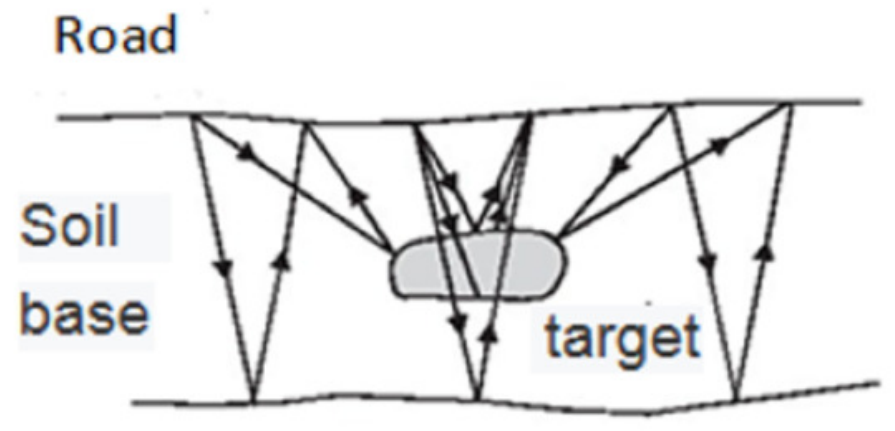

A

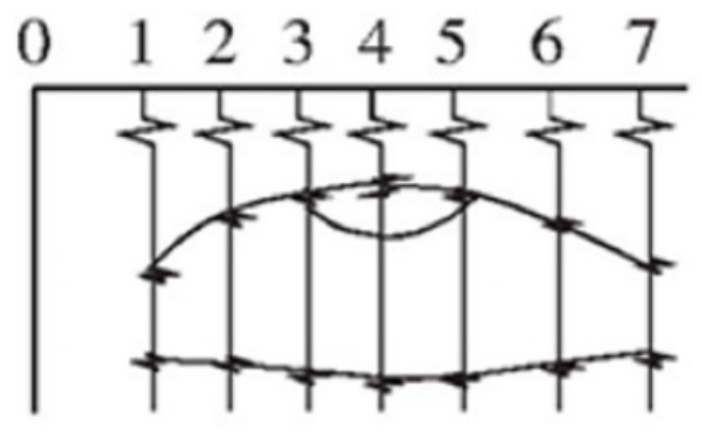

B 


\section{Figure 2}

Image segmentation using the Canny operator.

(A) Original image. (B) Gauss filter image. (C) Canny operator processing.
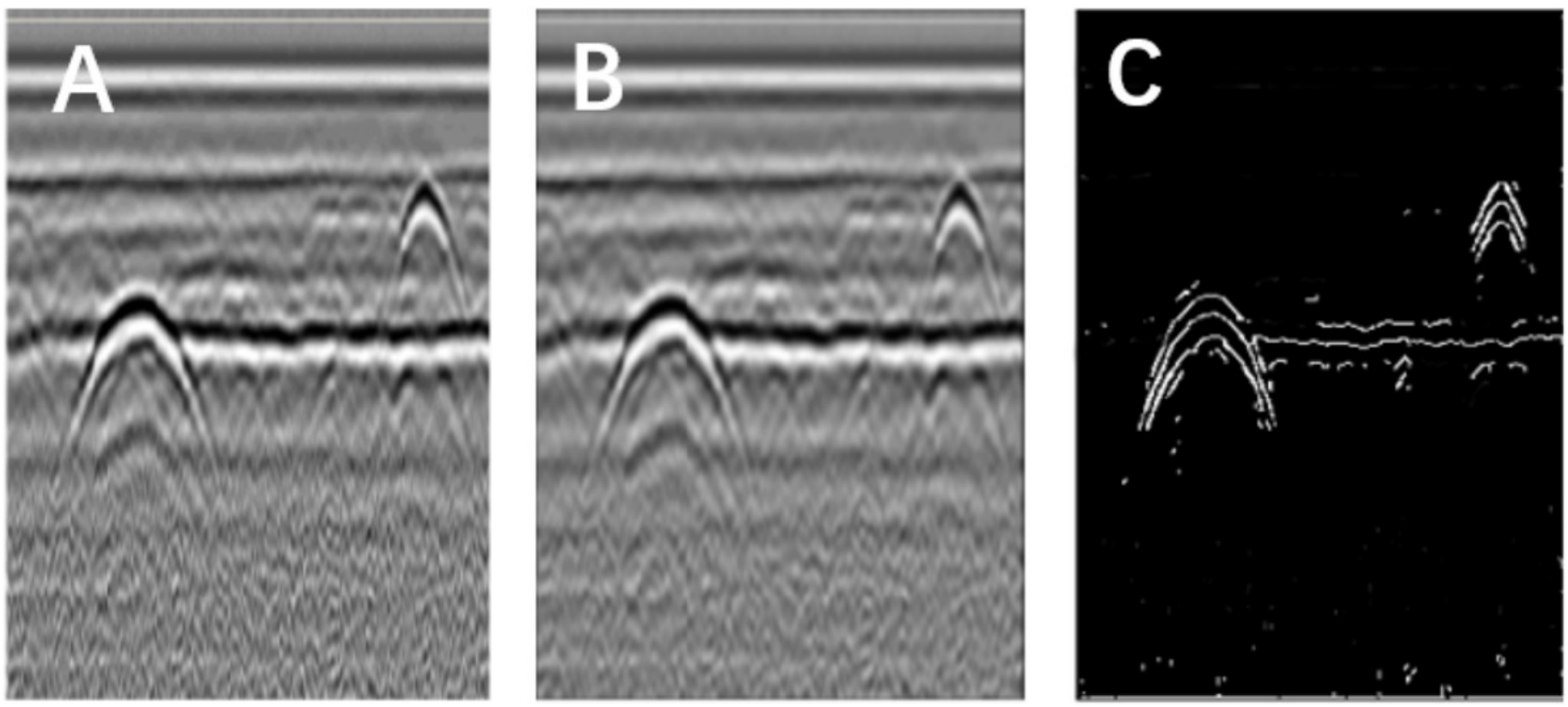


\section{Figure 3}

Morphological processing of digital images.

(A) Results obtained using the Canny operator. (B) Results obtained using morphological processing.
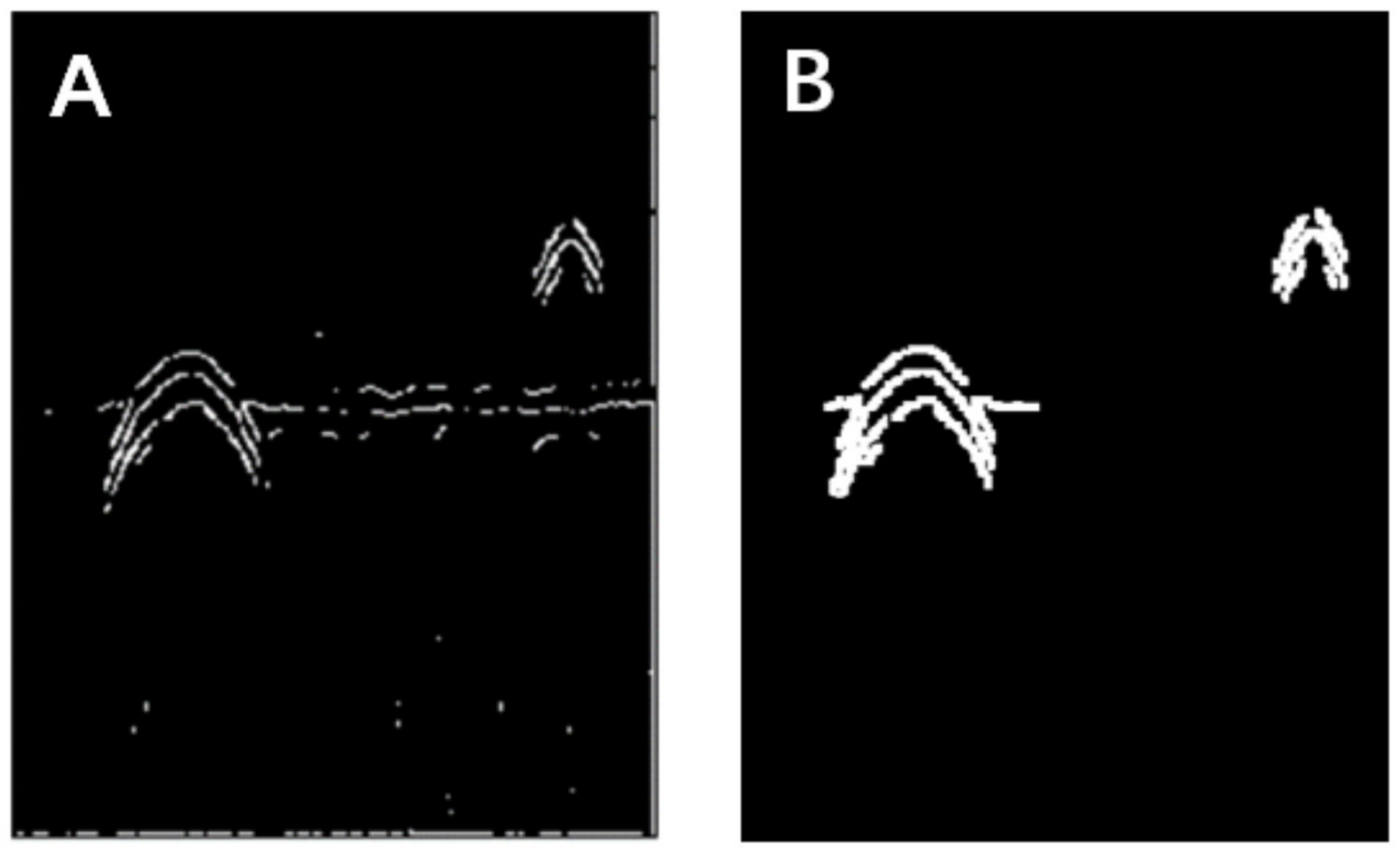
Figure 4

Projection segmentation results. 

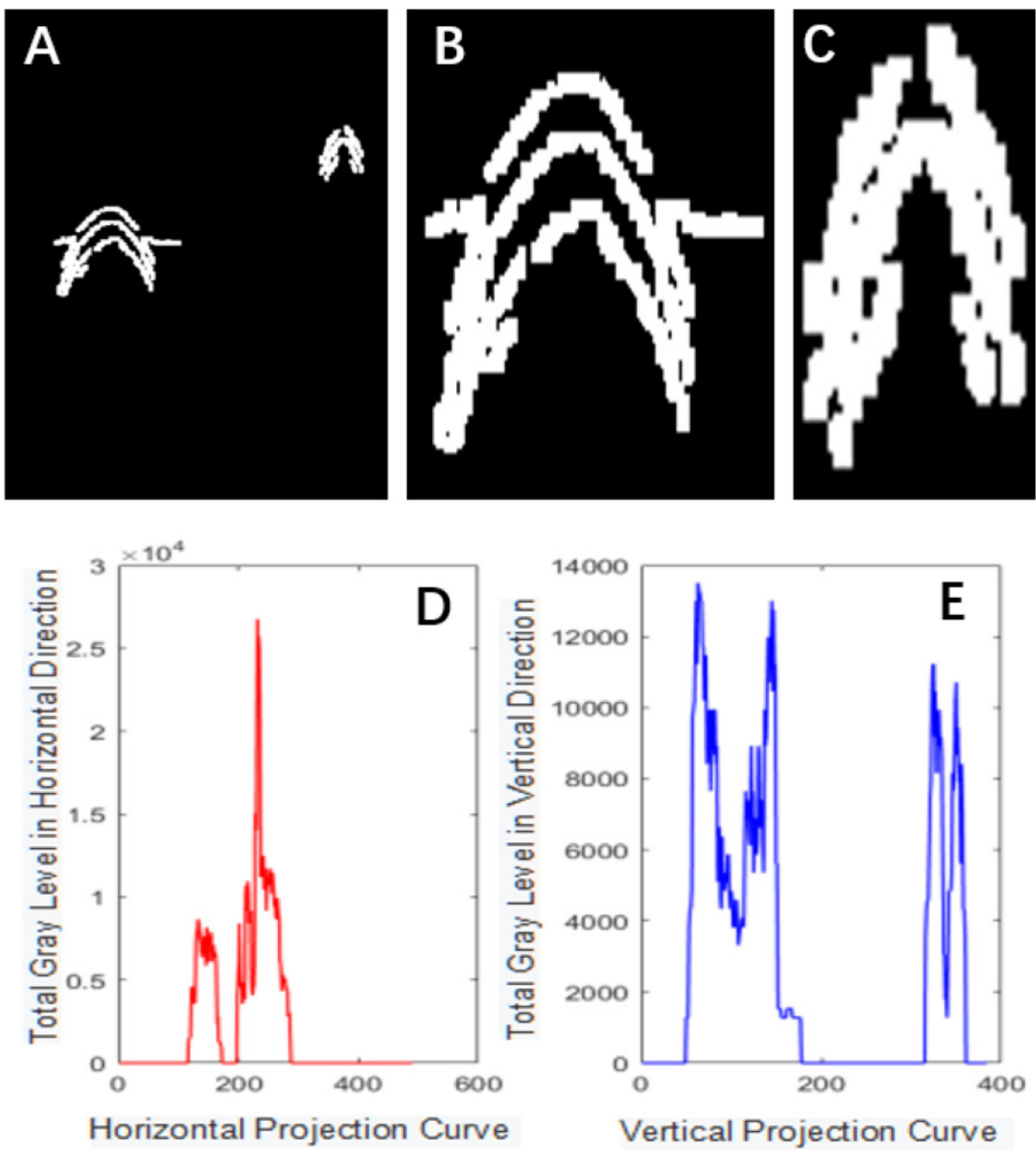
Figure 5

Dependence of the recognition rate on the number of features.

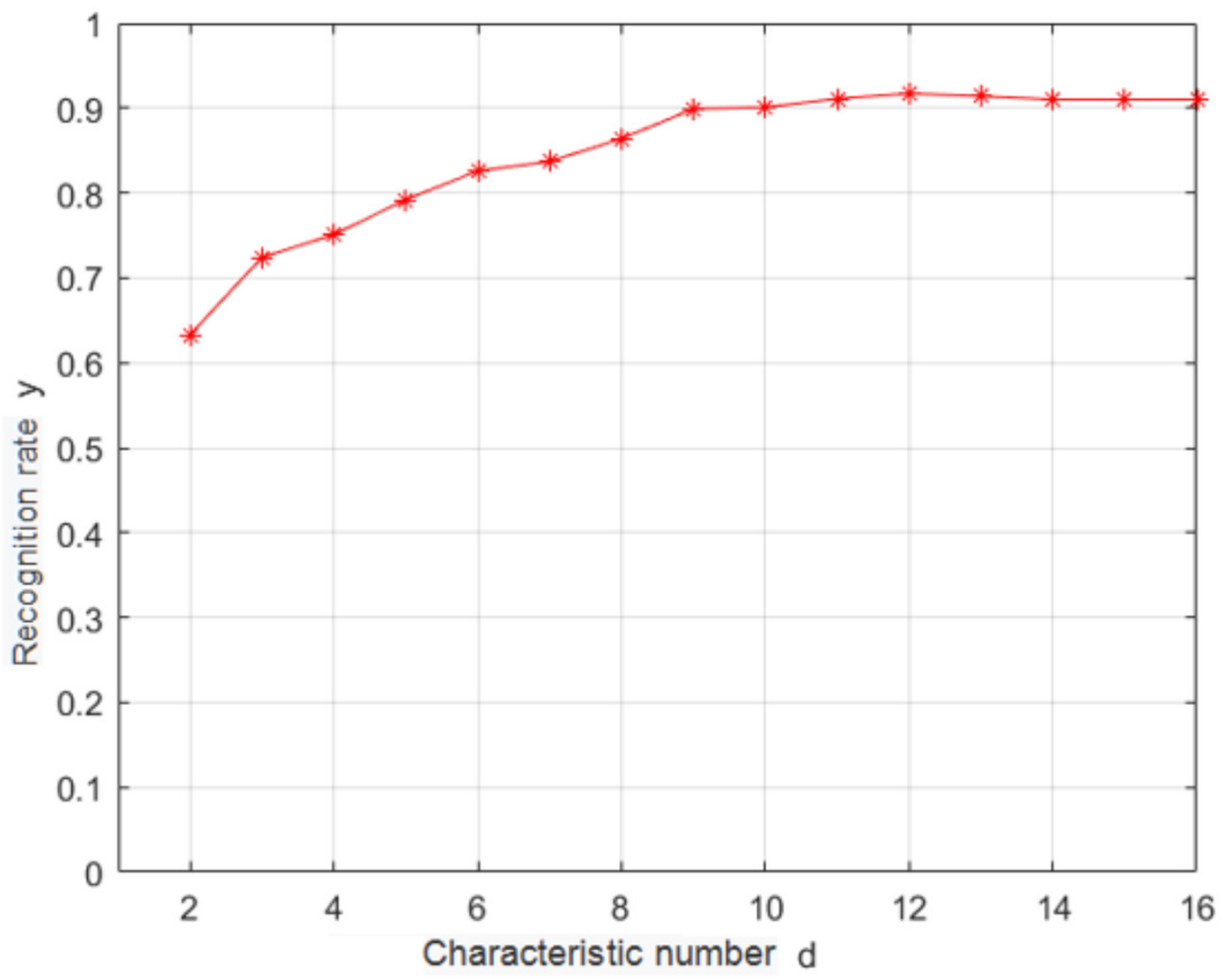


Figure 6

Parameter optimization results of the grid search method.

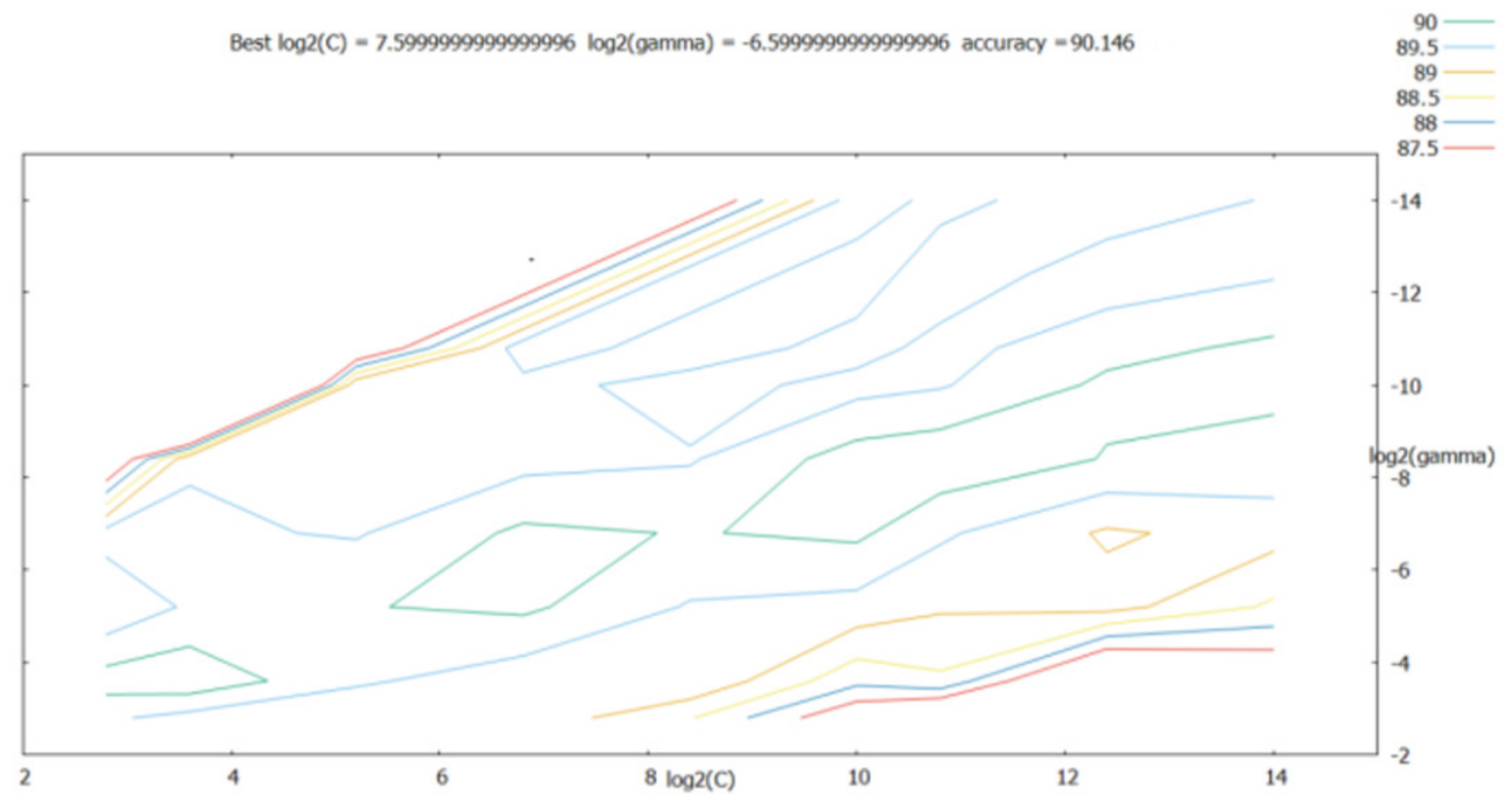


Figure 7

Results obtained after refining the step size.

Best $\log 2(C)=7.000000000000002 \log 2($ gamma $)=-5.200000000000001$ accuracy $=90.17$

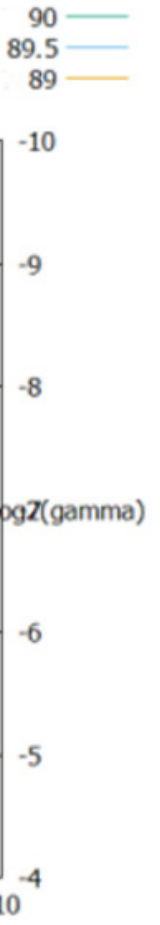


Figure 8

Test-set classification results after the grid search optimization of the SVM classifier parameters.

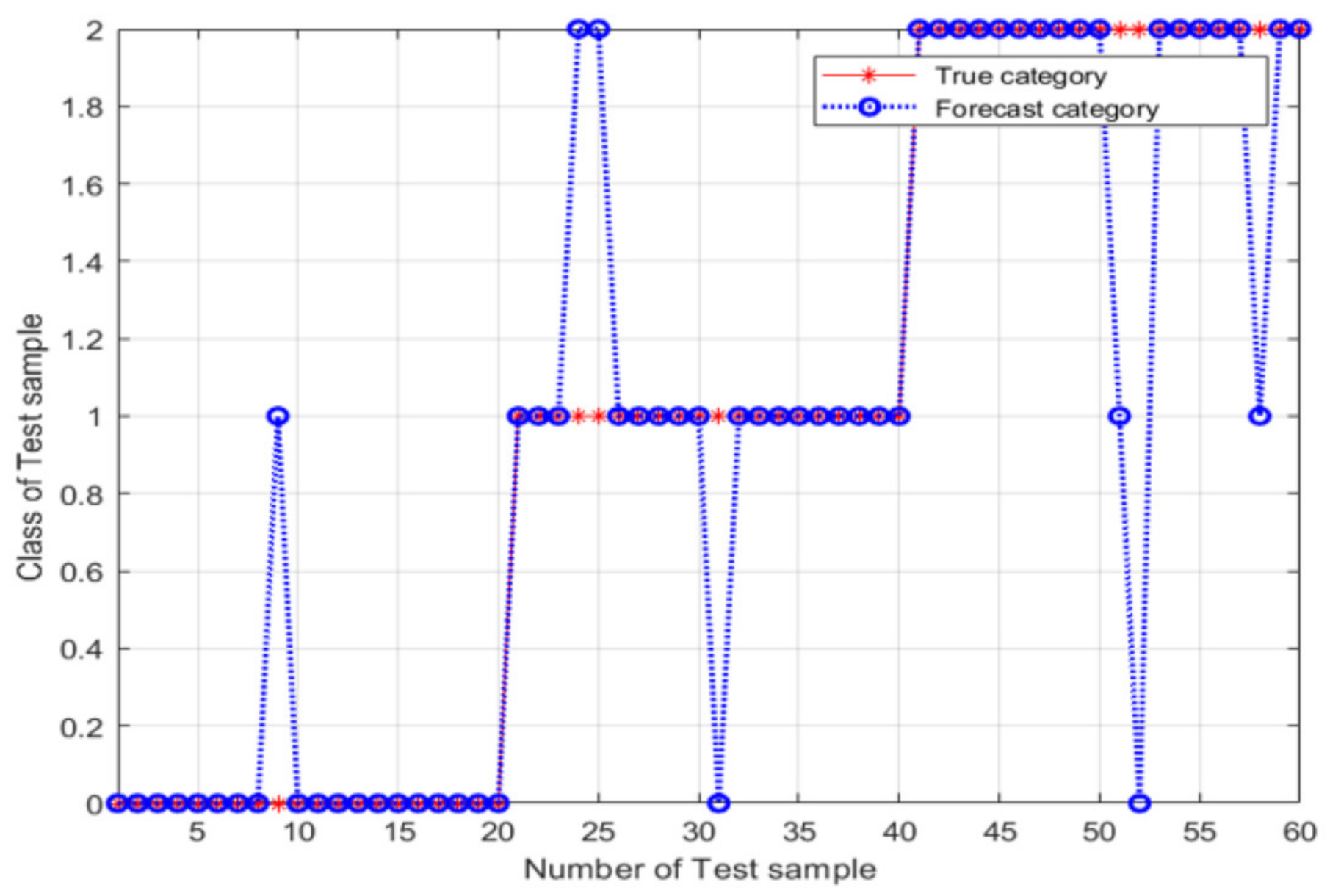


Figure 9

Fitness curve obtained after the PSO.

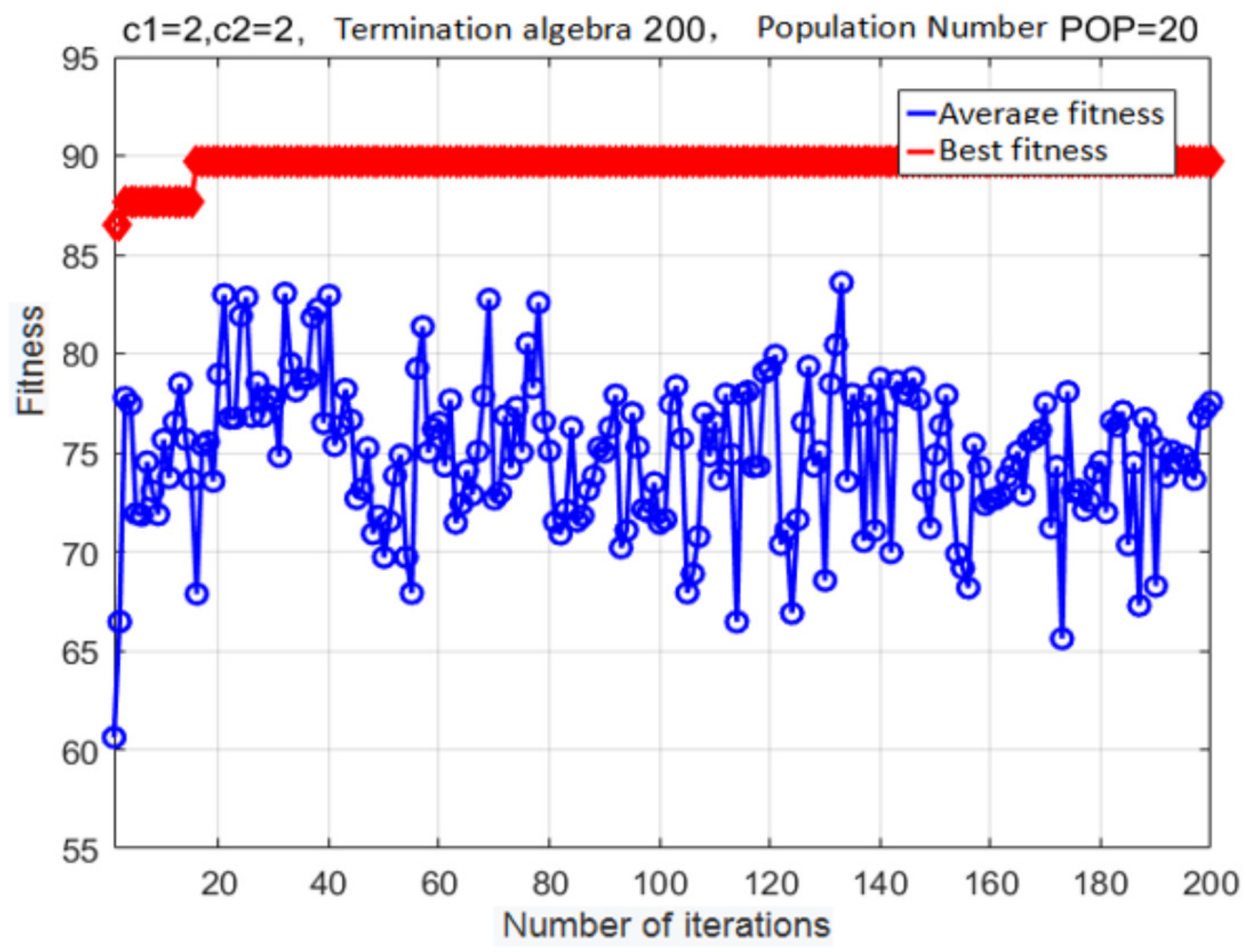


Figure 10

Fitness curve obtained after the variant PSO.

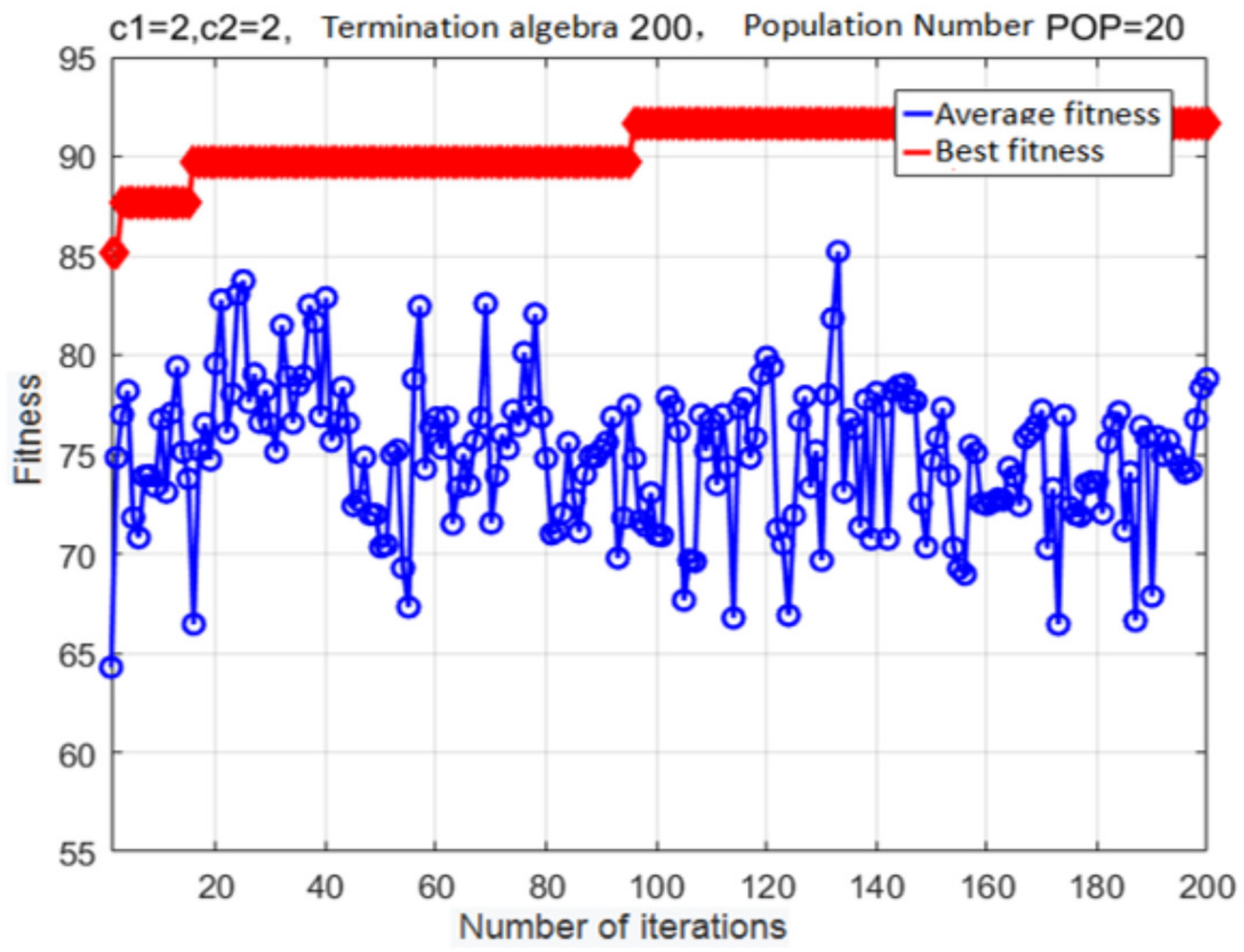


Figure 11

Validation results of the SVM model, for conventional PSO.

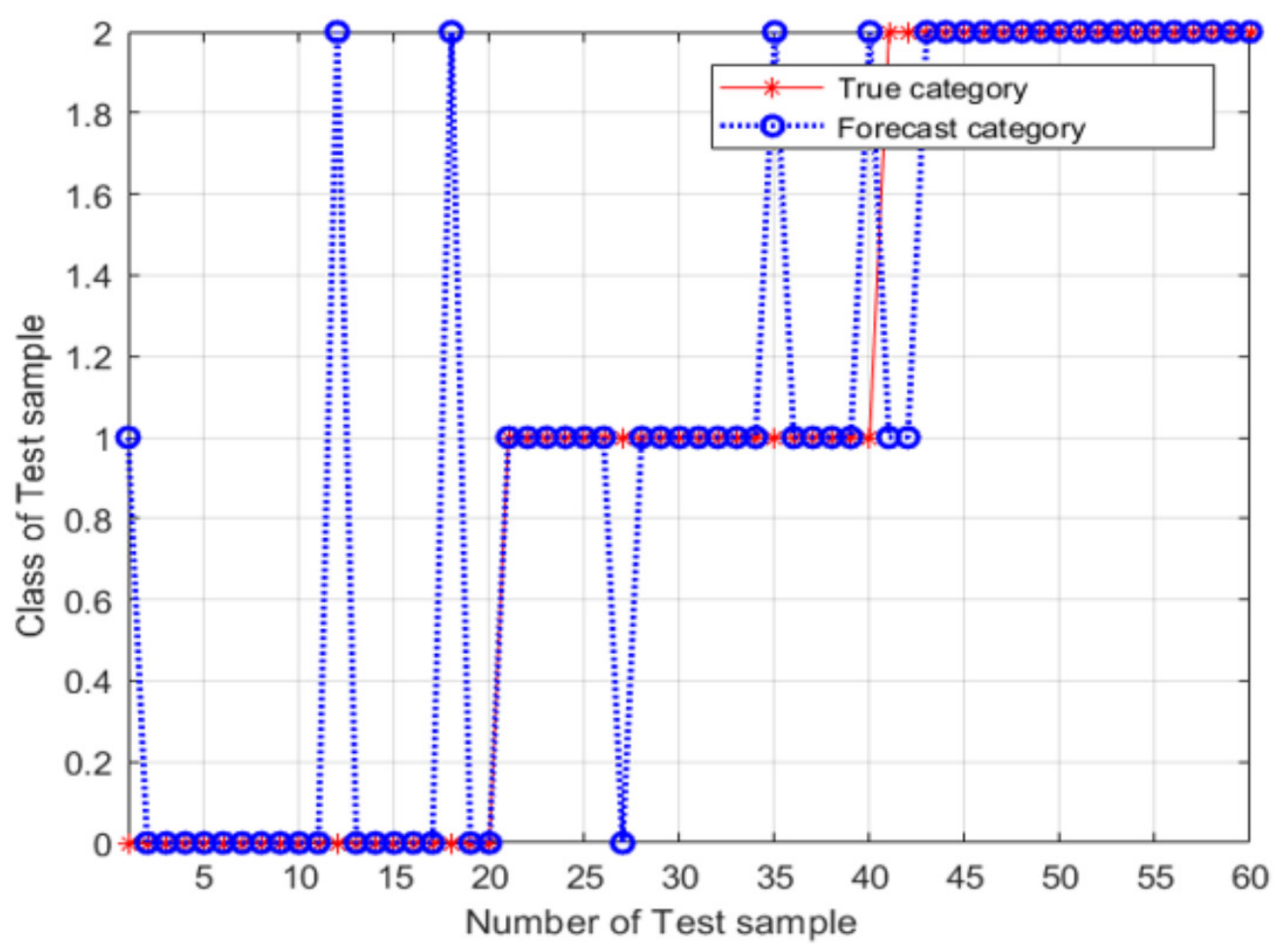


Figure 12

Validation results of the SVM model, for variant PSO.

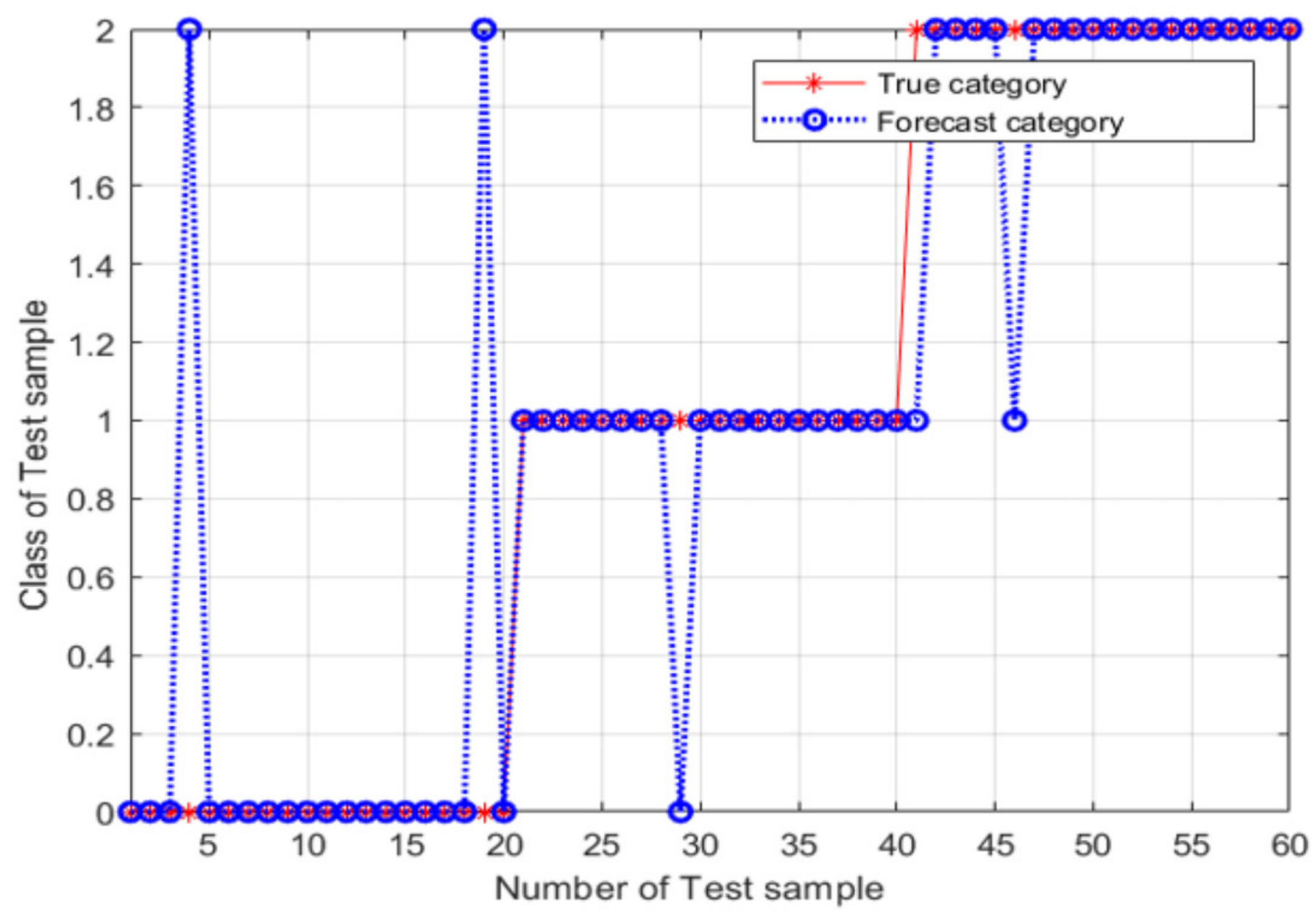

